\title{
Time-dependent fluorescence in nanoconfined solvents: Linear-response approximations and Gaussian statistics
}

\author{
Brian B. Laird ${ }^{\text {a) }}$ and Ward H. Thompson ${ }^{\text {b) }}$ \\ Department of Chemistry, University of Kansas, Lawrence, Kansas 66045, USA
}

(Received 8 March 2011; accepted 1 August 2011; published online 26 August 2011)

\begin{abstract}
The time-dependent fluorescence of a model dye molecule in a nanoconfined solvent is used to test approximations based on the dynamic and static linear-response theories and the assumption of Gaussian statistics. Specifically, the results of nonequilibrium molecular-dynamics simulations are compared to approximate expressions involving time correlation functions obtained from equilibrium simulations. Solvation dynamics of a model diatomic dye molecule dissolved in acetonitrile confined in a spherical hydrophobic cavity of radius 12,15 , and $20 \AA$ is used as the test case. Both the timedependent fluorescence energy, expressed as the normalized dynamic Stokes shift, and the timedependent position of the dye molecule after excitation are examined. While the dynamic linearresponse approximation fails to describe key aspects of the solvation dynamics, assuming Gaussian statistics reproduces the full nonequilibrium simulations well. The implications of these results are discussed. () 2011 American Institute of Physics. [doi:10.1063/1.3626825]
\end{abstract}

\section{INTRODUCTION}

Linear-response theories are widely and successfully used to describe a variety of condensed phase dynamics. These approaches describe the effect of some perturbation on nonequilibrium dynamics in terms of equilibrium fluctuations. In some circumstances (e.g., solvation dynamics) they have been sufficiently accurate that it is not uncommon for computational studies to report only the linear-response results, omitting nonequilibrium simulations entirely. However, breakdowns in linear response have been found in a number of different contexts.

One of these involves the solvation of neutral sodium atoms in tetrahydrofuran (THF). Schwartz and co-workers carried out a series of studies in which they investigated the solvation of a neutral $\mathrm{Na}$ atom prepared in liquid THF using two approaches. ${ }^{1-7}$ In one they generated the sodium atom by removing an electron from $\mathrm{Na}^{-}$while in the other they obtained it by adding an electron to $\mathrm{Na}^{+}$. If the system obeys linear response, the two experiments should display relaxation on the same time scale because the dynamics in both cases occur on different parts of the same underlying free energy surface. However, Bragg et al. instead observed that the solvation dynamics initiated from $\mathrm{Na}^{-}$are significantly slower than those started from $\mathrm{Na}^{+} .3$

Rotational energy relaxation is another notable example of a failure of the linear-response approximation. Moskun et al. examined the energy dissipation in highly rotationally excited $\mathrm{CN}$ dissolved in ethanol. ${ }^{8}$ They observed evidence for coherent rotational motion at short times indicating largeangle rotations, at odds with the traditional view of rotational diffusion in liquids. Molecular-dynamics (MD) simulations of $\mathrm{CN}$ in liquid argon reproduced the key features of the rotational coherence, but also showed strong deviations from

\footnotetext{
${ }^{a)}$ Electronic mail: blaird@ku.edu.

${ }^{b)}$ Electronic mail: wthompson@ku.edu.
}

linear-response behavior. ${ }^{8,9}$ By a detailed examination of the trajectories, it was possible to identify a distinct transition in dynamics when the $\mathrm{CN}$ rotor pushed an argon atom out of the first solvation shell. The dynamics prior to this event are well described by linear response while those following it are not. A similar pattern is observed in simulations using an $\mathrm{OH}$ rotor. ${ }^{10}$

Time-dependent fluorescence (TDF) measurements of solvation dynamics are frequently described using linearresponse approximations. In these experiments a dye molecule is photoexcited, typically via a charge-transfer transition, and the resulting fluorescence from the excited electronic state is monitored as a function of time after excitation. The Stokes shift or fluorescence energy at time $t, \Delta E(t)$, provides insight into the rearrangements of the solvent around the dye molecule in response to the change in its charge distribution. The result is often presented as the normalized dynamic Stokes shift,

$$
S(t)=\frac{\langle\Delta E(t)\rangle-\langle\Delta E(\infty)\rangle}{\langle\Delta E(0)\rangle-\langle\Delta E(\infty)\rangle}
$$

where $\langle\cdot\rangle$ indicates a statistical average and $\Delta E(\infty)$ is the relaxed Stokes shift. It is apparent in the description of the experiment that $S(t)$ can be simulated from nonequilibrium molecular dynamics. A dye molecule solute is equilibrated in its ground electronic state and then promoted suddenly to its excited state with an accompanying change in the charge distribution. Following this, $\Delta E(t)=E_{e}(t)-E_{g}(t)$ can be obtained from the difference in the excited- $(e)$ and ground- $(g)$ state dye-solvent interactions.

The dynamic linear-response approximation ${ }^{11-13}$ provides a route to $S(t)$ using only equilibrium dynamics, i.e., in terms of a correlation function of the equilibrium fluctuations of $\Delta E$ in the ground state, $C_{g}(t)$ $\equiv\langle\delta \Delta E(t) \delta \Delta E(0)\rangle_{g} /\left\langle\delta \Delta E(0)^{2}\right\rangle_{g}$; see Eq. (29) below. This approach has been successfully applied to solvation dynamics 
in many cases. Indeed, simulations of solvation dynamics are often carried out with this approximation. At the same time, solvation dynamics are an important testing ground for these approximations and violations of linear response have been observed. ${ }^{14-19}$

We recently showed ${ }^{20}$ that if one assumes $\Delta E$ is a Gaussian random variable, then $S(t)$ is equal to the equilibrium excited-state correlation function, $C_{e}(t)$ $\equiv\langle\delta \Delta E(t) \delta \Delta E(0)\rangle_{e} /\left\langle\delta \Delta E(0)^{2}\right\rangle_{e}$; see Eq. (33). This is the same result obtained using the static linear-response approximation $^{11,13,21}$ (also known as Onsager's regression hypothesis), which assumes that fluctuations away from excited-state equilibrium are small (see Sec. II B). The assumption of Gaussian statistics does not require this latter approximation and is therefore applicable to systems initiated far from equilibrium.

The connection between linear response and Gaussian statistics has been widely recognized, even if not always explicitly clear. In a sense, all three approximations just described - dynamic linear response, static linear response, and Gaussian statistics - can be thought of as "linear-response" theories. That is, they all describe a nonequilibrium response to a perturbation in terms of correlation functions that are linear in the perturbation. However, the three approaches do differ, both in the mathematical approximations invoked and the resulting physical implications. These differences are the focus of this paper in which the three approximations are compared in the context of a system for which they give distinguishable results. Thus, while we will see that the dynamic and static linear-response approximations display shortcomings, these are primarily due to the assumptions of small perturbations and the response can still be linear in the perturbation, even far from equilibrium, if the system exhibits Gaussian statistics.

Linear response and Gaussian statistics have been examined in the context of solvation dynamics by numerous groups. Fonseca and Ladanyi used MD simulations to study the accuracy of linear response for TDF of model diatomic dye molecules in methanol. ${ }^{14,15}$ They considered two models for the electronic transitions. In the first a dipole is created on the molecule in the excited electronic state starting from a nonpolar ground state, while in the second the molecule experiences a "dipole flip" upon excitation (by a switching of the charges on the atoms). The latter model is particularly interesting in that the equilibrium dynamics in the ground and excited state are the same so that all three approximations are tested by a single equilibrium simulation. They observed significant deviations from the linear-response and Gaussian statistics predictions in both cases. They attributed this to the hydrogen bonds between the solute dye and the methanol solvent which must be disrupted after excitation. The inertial dynamics that precede this disruption are well-described by dynamic linear response and they note that linear response appears to work well for solvents like water and acetonitrile because they have large inertial components, while methanol does not. ${ }^{14}$ A similar conclusion was reached by Turi et al. in mixed quantum-classical MD simulations of an excess electron in methanol. ${ }^{22}$ Namely, they also observed a failure of ground- or excited-state equilibrium time correlation func- tions to reproduce the nonequilibrium result and attributed it to the involvement of structural rearrangements of the solvent hydrogen bonds in the solvation response.

A failure of the dynamic linear-response approximation is also found when the electronic transition of the chromophore involves changes in size. Aherne et al. used MD simulations to investigate the case of a solute composed of a single Lennard-Jones atom, which can also carry charge, dissolved in water. ${ }^{18}$ When this solute underwent a transition that increased or decreased its radius, linear response failed to describe the solvation dynamics as represented by $S(t)$. It is interesting to note that this breakdown of linear response extends to even very short times. It thus includes the inertial response and cannot be attributed to rearrangements in the solvent hydrogen bonding patterns. Rather, it was attributed to the short-range interactions of the solute with a few, immediately adjacent solvent molecules, which do not explore at equilibrium the critical motions involved in the nonequilibrium response associated with a change in the solute size. ${ }^{18}$

Geissler and Chandler examined the dipole flip model and computed the (nonequilibrium) distribution of excitedand ground-state energy gaps at different times after excitation. ${ }^{19}$ They found that these distributions are Gaussian at all times, but depend on time, i.e., the energy gap obeys Gaussian, but nonstationary, statistics. The nonstationarity is a consequence of the changes in the hydrogen bonds between the dye and solvent. This nonstationarity may be at the root of the breakdown in the linear-response approximation observed for changes in solute size upon excitation. ${ }^{19}$

In this paper, the accuracy of the dynamic and static linear-response approximations and an assumption of Gaussian statistics for the relevant variables in a TDF measurement are examined for a model nanoconfined solvent. The solvation dynamics for this system represent an important test of these approximations as they display multiple time scales due to different equilibrium positions of the dye molecule in its ground and excited electronic states. The remainder of this paper is organized as follows. The linear response and Gaussian statistics approximations are reviewed in general form and in application to a TDF measurement in Sec. II. The nanoconfined solvent system and the simulations used in this work are described in detail in Sec. III. The results of nonequilibrium MD simulations and the equilibrium MDbased linear-response and Gaussian statistics approximations are presented, compared, and discussed in Sec. IV. Finally, some concluding remarks are offered in Sec. V.

\section{THEORY}

In this section, the linear-response and Gaussian statistics approximations are outlined for the case of a chromophore electronically excited in a condensed phase environment. However, the results can be straightforwardly generalized to other cases.

While a common experimental situation involves monitoring the fluorescence energy as a function of time after excitation, expressions for the change in an arbitrary observable after excitation are considered first. We consider the change in some property, $A$, of the chromophore/solvent system after 
excitation of the chromophore. This can be expressed as

$$
\begin{aligned}
\langle A(t)\rangle_{n e} & =\frac{\int d \mathbf{p} \int d \mathbf{q} A(t) e^{-\beta H_{g}}}{\int d \mathbf{p} \int d \mathbf{q} e^{-\beta H_{g}}}, \\
& =\frac{\int d \mathbf{p} \int d \mathbf{q}\left[e^{\hat{L}_{e} t} A(0)\right] e^{-\beta H_{g}}}{\int d \mathbf{p} \int d \mathbf{q} e^{-\beta H_{g}}},
\end{aligned}
$$

where $H_{g}\left(H_{e}\right)$ is the ground- (excited-) state Hamiltonian and $\hat{L}_{e}$ is the excited-state Liouville operator, $\hat{L}_{e}=\left\{H_{e}, \cdot\right\}$, where $\{F, G\}=\sum_{j}\left[\left(\partial F / \partial p_{j}\right)\left(\partial G / \partial q_{j}\right)-\left(\partial F / \partial q_{j}\right)\left(\partial G / \partial p_{j}\right)\right]$ indicates a Poisson bracket. From Eq. (2) it is clear that the nonequilibrium behavior of $A$ as a function of time is determined by excited-state dynamics which are initiated from configurations and momenta sampled from the ground-state chromophore system. The quantity $\langle A(t)\rangle_{n e}$ can be obtained from nonequilibrium MD simulations in the fashion indicated by this equation: excited-state dynamics begun from conditions obtained from an equilibrated ground-state system. However, linear response-type assumptions are frequently invoked, which approximate $\langle A(t)\rangle_{n e}$ by equilibrium time correlation functions. In the remainder of this section, we outline the nature of a dynamic linear-response approximation and an assumption that the relevant variables exhibit Gaussian statistics. The latter is also compared to the static linear-response approach, which provides the same expression for $\langle A(t)\rangle_{n e}$ based on a different approximation.

\section{A. Dynamic linear-response approximation}

In the dynamic linear-response approximation, the nonequilibrium behavior of a variable $A$ can be approximated by results from equilibrium molecular-dynamics simulations. Here we summarize this well-established approach ${ }^{11,13}$ for completeness. The general approach is to write the Hamiltonian for the system as a zeroth-order Hamiltonian and a "perturbation" that is turned on at the moment of excitation, taken to be $t=0$,

$$
H(t)=H_{g}+\theta(t)\left[H_{e}-H_{g}\right]=H_{g}+\theta(t) \Delta E,
$$

where $\theta(t)$ is the Heaviside step function and, $H_{g}$ and $H_{e}$ are the Hamiltonians for the ground and excited state dye molecule, respectively, and the second equality uses the definition of the energy gap, which is the same as the fluorescence energy,

$$
\Delta E=H_{e}-H_{g} .
$$

Then, the Liouville operator can be written for all times as

$$
\hat{L}=\hat{L}_{g}+\hat{L}_{\Delta} \theta(t),
$$

where $\hat{L}_{g}=\left\{H_{g}, \cdot\right\}$ is the Liouville operator for the ground state and $\hat{L}_{\Delta}=\{\Delta E, \cdot\}$ is that associated with the change due to excitation; note that $\hat{L}=\hat{L}_{e}$ for $t \geq 0$. If we note that Eq. (2) can be alternately written with the time evolution on the distribution function,

$$
\begin{aligned}
\langle A(t)\rangle_{n e} & =\int d \mathbf{p} \int d \mathbf{q} A(0) e^{-\hat{L}\left(t+t_{0}\right)} f_{e q}(\mathbf{p}, \mathbf{q}) \\
& =\int d \mathbf{p} \int d \mathbf{q} A(0) f(\mathbf{p}, \mathbf{q}, t),
\end{aligned}
$$

a linear-response approximation can be substituted for $f(\mathbf{p}, \mathbf{q}, t)$. In this equation, it is assumed the system is at equilibrium at time $-t_{0}$ with a distribution function, $f_{e q}$ $=e^{-\beta H_{g}} / Q$, which evolves under $H(t)$ to $f(\mathbf{p}, \mathbf{q}, t)$ at time $t$.

In the dynamic linear-response approximation, the distribution function is expanded in powers of the perturbation, truncated at the linear term: $f \simeq f_{0}+f_{1}$. Then,

$\frac{\partial f}{\partial t}=\frac{\partial f_{0}}{\partial t}+\frac{\partial f_{1}}{\partial t}=-\hat{L} f=-\hat{L}_{g} f_{0}-\hat{L}_{g} f_{1}-\hat{L}_{\Delta} f_{0} \theta(t)$,

where terms second-order in the perturbation have been neglected. Upon setting equal the different orders of the perturbation this gives two equations, with the solutions ${ }^{13}$

$$
\begin{gathered}
f_{0}(\mathbf{p}, \mathbf{q}, t)=e^{-\hat{L}_{g}\left(t+t_{0}\right)} f_{e q}(\mathbf{p}, \mathbf{q})=f_{e q}(\mathbf{p}, \mathbf{q}) \\
f_{1}(\mathbf{p}, \mathbf{q}, t)=f_{1}\left(\mathbf{p}, \mathbf{q},-t_{0}\right)-\int_{-t_{0}}^{t} d s e^{-\hat{L}_{g}(t-s)} \hat{L}_{\Delta} f_{e q}(\mathbf{p}, \mathbf{q}) \theta(s) \\
=-\int_{0}^{t} d s e^{-\hat{L}_{g}(t-s)} \hat{L}_{\Delta} f_{e q}(\mathbf{p}, \mathbf{q})
\end{gathered}
$$

because $f_{e q}$ is unchanged under ground-state dynamics and $f_{1}=0$ before the excitation at $t=0$. Evaluating the Poisson bracket associated with $\hat{L}_{\Delta} f_{e q}(\mathbf{p}, \mathbf{q})$ gives ${ }^{13}$

$$
\hat{L}_{\Delta} f_{e q}(\mathbf{p}, \mathbf{q})=-\beta \Delta \dot{E} f_{e q}(\mathbf{p}, \mathbf{q})
$$

so that

$$
\begin{aligned}
\langle A(t)\rangle_{n e}= & \int d \mathbf{p} \int d \mathbf{q} A(0) f_{e q}(\mathbf{p}, \mathbf{q})+\beta \int d \mathbf{p} \int d \mathbf{q} A(0) \\
& \times \int_{0}^{t} d s e^{-\hat{L}_{g}(t-s)} \Delta \dot{E} f_{e q}(\mathbf{p}, \mathbf{q}) \\
= & \langle A\rangle_{g}+\beta \int_{0}^{t} d s \int d \mathbf{p} \\
& \times \int d \mathbf{q} A(0) e^{-\hat{L}_{g}(t-s)} \dot{\Delta E} f_{e q}(\mathbf{p}, \mathbf{q}) \\
= & \langle A\rangle_{g}+\beta \int_{0}^{t} d s\langle A(t-s) \Delta \dot{E}(0)\rangle_{g} .
\end{aligned}
$$

This can be rearranged, using the properties of the correlation function, ${ }^{23}$ as

$$
\langle A(t)\rangle_{n e}=\langle A\rangle_{g}+\beta\left[\langle A(t) \Delta E(0)\rangle_{g}-\langle A(0) \Delta E(0)\rangle_{g}\right] .
$$

\section{B. Assumption of Gaussian statistics}

As we have shown previously, ${ }^{20}$ the assumption that a property of interest $A$ and the energy gap, $\Delta E$, defined in 
Eq. (4) are Gaussian random variables provides another approach to obtaining nonequilibrium properties from equilibrium simulations. Here we outline this approximation.

The nonequilibrium response of $A$ to a change in chromophore electronic state given in Eq. (2) can be rewritten by noting that $H_{g}=H_{e}-\Delta E$. Thus, Eq. (2) can be rearranged as

$$
\langle A\rangle_{n e}(t)=\frac{\int d \mathbf{p} \int d \mathbf{q} A(t) e^{\beta \Delta E} e^{-\beta H_{e}}}{\int d \mathbf{p} \int d \mathbf{q} e^{\beta \Delta E} e^{-\beta H_{e}}},
$$

where the time-evolution of $A(t)$ occurs under the excited state Hamiltonian, $H_{e}$. Dividing the numerator and denominator by the excited state partition function gives

$$
\langle A\rangle_{n e}(t)=\frac{\left\langle A(t) e^{\beta \Delta E}\right\rangle_{e}}{\left\langle e^{\beta \Delta E}\right\rangle_{e}},
$$

where $\langle\cdot\rangle_{e}$ is an equilibrium excited state average. Defining the average energy gap in the excited state as $\overline{\Delta E} \equiv\langle\Delta E\rangle_{e}$, this can be rewritten as

$$
\langle A\rangle_{n e}(t)=\frac{\left\langle A(t) e^{\beta \delta \Delta E}\right\rangle_{e} e^{\beta \overline{\Delta E}}}{\left\langle e^{\beta \Delta E}\right\rangle_{e} e^{\beta \overline{\Delta E}}}=\frac{\left\langle A(t) e^{\beta \delta \Delta E}\right\rangle_{e}}{\left\langle e^{\beta \delta \Delta E}\right\rangle_{e}},
$$

where $\delta \Delta E(t)=\Delta E(t)-\overline{\Delta E}$. Moreover, defining $\bar{A}$ $\equiv\langle A\rangle_{e}$, this gives

$$
\begin{aligned}
\langle A\rangle_{n e}(t)-\langle A\rangle_{e} & =\frac{\left\langle A(t) e^{\beta \delta \Delta E}\right\rangle_{e}}{\left\langle e^{\beta \delta \Delta E}\right\rangle_{e}}-\frac{\bar{A}\left\langle e^{\beta \delta \Delta E}\right\rangle_{e}}{\left\langle e^{\beta \delta \Delta E}\right\rangle_{e}} \\
& =\frac{\left\langle\delta A(t) e^{\beta \delta \Delta E}\right\rangle_{e}}{\left\langle e^{\beta \delta \Delta E}\right\rangle_{e}},
\end{aligned}
$$

where $\delta A(t)=A(t)-\bar{A}$.

To this point, we have invoked no approximations. However, if we now assume that $A$ and $\Delta E$ are Gaussian random variables, we can derive an expression for $\langle A\rangle_{n e}(t)$ involving only equilibrium correlation functions. Specifically, the numerator can be written as

$$
\begin{aligned}
\left\langle\delta A(t) e^{\beta \delta \Delta E}\right\rangle_{e}= & \sum_{n=0}^{\infty} \frac{1}{n !} \beta^{n}\left\langle\delta A(t) \delta \Delta E(0)^{n}\right\rangle_{e}, \\
= & \sum_{n=0}^{\infty} \frac{1}{(2 n) !} \beta^{2 n}\left\langle\delta A(t) \delta \Delta E(0)^{2 n}\right\rangle_{e} \\
& +\sum_{n=0}^{\infty} \frac{1}{(2 n+1) !} \beta^{2 n+1}\left\langle\delta A(t) \delta \Delta E(0)^{2 n+1}\right\rangle_{e} .
\end{aligned}
$$

However, if $\delta A(t)$ and $\delta \Delta E(t)$ are Gaussian random variables with zero mean, then all $m$-point correlation functions $\left\langle\delta A\left(t_{1}\right) \delta \Delta E\left(t_{2}\right) \ldots \delta \Delta E\left(t_{m}\right)\right\rangle_{e}$ are equal zero if $m$ is odd and, if $m$ is even, to the sum of all possible factorizations of the correlation function in terms of two-point correlation functions, ${ }^{24}$ $\left\langle\delta A\left(t_{i}\right) \delta \Delta E\left(t_{j}\right)\right\rangle_{e}$, a property often referred to as Wick's theorem. ${ }^{25}$ That is, if $m$ is odd, as in the correlation functions $\left\langle\delta A(t) \delta \Delta E(0)^{2 n}\right\rangle_{e}$ where $m=2 n+1$, Wick's theorem says this can be reduced to two-point correlation functions, $e$.g., for $\left\langle\delta A(t) \delta \Delta E(0)^{2}\right\rangle_{e}$ these are $\langle\delta A(t) \delta \Delta E(0)\rangle_{e}\langle\delta \Delta E(0)\rangle_{e}$ and $\left\langle\delta \Delta E(0)^{2}\right\rangle_{e}\langle\delta A(t)\rangle_{e}$. Because $\delta \Delta E$ and $\delta A$ have zero mean,

$$
\left\langle\delta A(t) \delta \Delta E(0)^{2 n}\right\rangle_{e}=0,
$$

for all $n$. If $m$ is even, as in $\left\langle\delta A(t) \delta \Delta E(0)^{2 n+1}\right\rangle_{e}$ where $m$ $=2 n+2$, Wick's theorem says the correlation function can be reduced to products of two-point correlation functions, e.g., $\left\langle\delta A(t) \delta \Delta E(0)^{3}\right\rangle_{e} \propto\langle\delta A(t) \delta \Delta E(0)\rangle_{e}\left\langle\delta \Delta E(0)^{2}\right\rangle_{e}$. Accounting for the number of possible two-point correlation functions, this gives

$$
\left\langle\delta A(t) \delta \Delta E(0)^{2 n+1}\right\rangle_{e}=\frac{(2 n+1) !}{n ! 2^{n}}\langle\delta A(t) \delta \Delta E(0)\rangle_{e}\left\langle\delta \Delta E^{2}\right\rangle_{e}^{n} .
$$

Using these approximations gives Eq. (19) as

$$
\begin{aligned}
&\left\langle\delta A(t) e^{\beta \delta \Delta E}\right\rangle_{e} \simeq \beta\langle\delta A(t) \delta \Delta E(0)\rangle_{e} \\
& \times \sum_{n=0}^{\infty} \frac{1}{n !} \frac{\beta^{2 n}\left\langle\delta \Delta E^{2}\right\rangle_{e}^{n}}{2^{n}}, \\
& \simeq \beta\langle\delta A(t) \delta \Delta E(0)\rangle_{e} e^{\beta^{2}\left\langle\delta \Delta E^{2}\right\rangle_{e} / 2} .
\end{aligned}
$$

The denominator in Eq. (17) can be easily evaluated with the assumption of Gaussian statistics for $\delta \Delta E$. The average $\left\langle e^{\beta \delta \Delta E}\right\rangle_{e}$ is the characteristic function, evaluated at $-i \beta$, for the probability distribution for $\delta \Delta E$, which, if $\delta \Delta E$ is assumed to be a Gaussian random variable, is given by ${ }^{11}$

$$
\left\langle e^{\beta \delta \Delta E}\right\rangle_{e} \simeq e^{\beta^{2}\left\langle(\delta \Delta E)^{2}\right\rangle_{e} / 2},
$$

Then, combining Eqs. (23) and (24) gives the Gaussian statistics approximation for the nonequilibrium response,

$$
\langle A(t)\rangle_{n e}-\langle A\rangle_{e} \simeq \beta\langle\delta A(t) \delta \Delta E(0)\rangle_{e} .
$$

This result can be written in a form similar to Eq. (13) as

$$
\langle A(t)\rangle_{n e}-\langle A\rangle_{e} \simeq \beta\left[\langle A(t) \Delta E(0)\rangle_{e}-\langle A\rangle_{e}\langle\Delta E(0)\rangle_{e}\right] .
$$

\section{Static linear-response approximation}

The same result is obtained by the static linear-response approximation, or Onsager regression hypothesis. ${ }^{21,27-29}$ Specifically, beginning with Eq. (17) which contains no approximation, one can linearize the exponential,

$$
e^{\beta \delta \Delta E} \approx 1+\beta \delta \Delta E,
$$

in the numerator and denominator ${ }^{26}$ by assuming that $\beta \delta \Delta E \ll 1$, i.e., that the fluctuations from equilibrium are small on the scale of $k_{B} T$. This gives the same expression for $\langle A\rangle_{n e}(t)$ as obtained from assuming Gaussian statistics in Eqs. (25) and (26),

$$
\begin{aligned}
\langle A\rangle_{n e}(t)-\langle A\rangle_{e} & \simeq \frac{\langle\delta A(t)\rangle_{e}+\beta\langle\delta A(t) \delta \Delta E\rangle_{e}}{1+\beta\langle\delta \Delta E\rangle_{e}} \\
& =\beta\langle\delta A(t) \delta \Delta E\rangle_{e},
\end{aligned}
$$

because $\langle\delta A(t)\rangle_{e}=0$ and $\langle\delta \Delta E(t)\rangle_{e}=0$. While it provides the same end result, it is based on different assumptions. ${ }^{20}$ For example, it is possible to show (see the Appendix) that 
in the linear-response limit the results from the static and dynamic approaches are equivalent. ${ }^{43}$ Further, the static linearresponse approximation takes all higher order correlation functions to be zero, $\left\langle\delta A(t) \delta \Delta E(0)^{n}\right\rangle_{e}=0$ for $n>1$, while the assumption of Gaussian statistics indicates those correlation functions are not zero, but do not provide any new information about the nonequilibrium time dependence of $A$. The former, which relies on the assumption of small fluctuations from equilibrium, is generally not a reasonable assumption, while the latter can be (see below). These approximations for the higher-order correlation functions need not merely be an academic issue, as they can potentially be used as diagnostics for the quality of the Gaussian statistics approximation. Moreover, because the Gaussian statistics and static linear-response results for $\langle A\rangle_{n e}(t)$ are the same, it is reasonable to ask which physical assumption is valid when the approximation is accurate. It has long been recognized that linear-response approximations such as Eq. (28) can agree with nonequilibrium simulation results even for responses initiated far from equilibrium. ${ }^{32}$ This indicates that the Gaussian statistics approximation is more general and should be thought of as the underlying physical and mathematical assumption for Eq. (28), an issue that is addressed further in Sec. IV. It is important to note, however, that this does not indicate a breakdown of linear response as the assumption of Gaussian statistics actually suggests a global linear response whereas the static linear-response approximation expressed in Eq. (27) implies only a local one.

\section{Application to time-dependent fluorescence}

For the case of time-dependent fluorescence in nanoconfined solvents considered in this paper, we are primarily interested in the nonequilibrium response of two variables: the ground-to-excited-state energy gap, $\Delta E(t)$, and the position of the chromophore expressed as the distance from the confining framework wall, $d(t)$.

\section{Dynamic linear-response approximations}

It is straightforward to show that for $A=\Delta E$, the dynamic linear-response approximation for the normalized dynamic Stokes shift, Eq. (1), gives

$$
S(t) \simeq C_{g}(t)=\frac{\langle\delta \Delta E(t) \delta \Delta E(0)\rangle_{g}}{\left\langle\delta \Delta E(0)^{2}\right\rangle_{g}},
$$

where $\delta \Delta E(t)=\Delta E(t)-\langle\Delta E\rangle_{g}$. If we are instead concerned about the chromophore position, its change after excitation can be expressed as

$$
\langle\Delta d(t)\rangle_{n e} \equiv\langle d(t)\rangle_{n e}-\langle d(0)\rangle_{n e}=\langle d(t)\rangle_{n e}-\langle d\rangle_{g} .
$$

Then, if $A=d$ the dynamic linear response result, Eq. (13), gives

$$
\langle\Delta d(t)\rangle_{n e} \simeq \beta\left[\langle d(t) \Delta E(0)\rangle_{g}-\langle d(0) \Delta E(0)\rangle_{g}\right]
$$

\section{Gaussian statistics approximations}

Assuming Gaussian statistics for the ground-to-excitedstate energy gap gives Eq. (25) as

$$
\langle\Delta E(t)\rangle_{n e}-\langle\Delta E\rangle_{e} \simeq \beta\langle\delta \Delta E(t) \delta \Delta E(0)\rangle_{e},
$$

with $\delta \Delta E(t)=\Delta E(t)-\langle\Delta E\rangle_{e}$ for this excited-state average. The normalized dynamics Stokes shift, $S(t)$, is then given by

$$
S(t) \simeq C_{e}(t)=\frac{\langle\delta \Delta E(t) \delta \Delta E(0)\rangle_{e}}{\left\langle\delta \Delta E^{2}\right\rangle_{e}} .
$$

Here, we have used the facts that $\langle\Delta E(0)\rangle_{n e}=\langle\Delta E\rangle_{g}$ and $\langle\Delta E(\infty)\rangle_{n e}=\langle\Delta E\rangle_{e}$. If we are instead concerned about the chromophore position, its change after excitation can be expressed, assuming that both $\delta d$ and $\delta \Delta E$ are Gaussian random variables, as

$$
\langle\Delta d(t)\rangle_{n e} \simeq \beta\left[\langle\delta d(t) \delta \Delta E(0)\rangle_{e}-\langle\delta d(0) \delta \Delta E(0)\rangle_{e}\right],
$$

where $\delta d(t)=d(t)-\langle d\rangle_{g}$.

\section{SIMULATION DETAILS}

Equilibrium and nonequilibrium simulations of a solute dissolved in a solvent confined inside a spherical nanocavity have been carried out. The solute is a model diatomic molecule (hereafter denoted as AB) with Lennard-Jones and Coulombic interactions. The details of the model are the same as those in Refs. 30 and 31. Briefly, the A and B LennardJones parameters are the same and are independent of electronic state. The two electronic states are related by a chargetransfer transition $\left(\mu_{g r}=1.44 \mathrm{D}, \mu_{e x}=7.2 \mathrm{D}\right)$ with the excited state $2 \mathrm{eV}$ higher in energy than the ground state. Although a two valence bond state model $^{33}$ is used, the electronic coupling is sufficiently small $(0.01 \mathrm{eV})$ that these simulations involve effectively fixed charges in the two electronic states. Simulations have been carried out with a $\mathrm{CH}_{3} \mathrm{CN}$ solvent using the three-site rigid molecule model of Jorgensen and Briggs. ${ }^{34}$ In this model the methyl group is treated as a "unified atom."

The interactions of the solute and solvent molecules with the cavity walls involve only Lennard-Jones interactions. $^{30,31,35,36}$ The potential depends only on the radial distance of the Lennard-Jones site on the molecule from the center of the cavity. We consider a single solvent density, $\rho=0.7 \mathrm{~g} / \mathrm{cm}^{3}$ (the bulk density of $\mathrm{CH}_{3} \mathrm{CN}$ is $\left.0.786 \mathrm{~g} / \mathrm{cm}^{3}\right) .{ }^{37}$ The density of the solution inside the cavity is taken to be approximately the same for a given solvent as the cavity size is varied. ${ }^{38}$ The cavity radius, $R_{\text {cav }}$, is taken to be 12,15 , and $20 \AA$.

Three types of MD simulations were carried out: (1) equilibrium simulations with the solute in the ground state, (2) equilibrium simulations with the solute in the excited state, (3) nonequilibrium simulations in which the solute is promoted from the ground to the excited state and the subsequent dynamics followed. The first two simulations and the ground state equilibration for the third were performed in the NVT ensemble using a Nosé-Poincaré thermostat at $290 \mathrm{~K}$, which should not introduce any significant error into the time 
correlation functions. ${ }^{39}$ A generalized leapfrog integrator ${ }^{40}$ was used with a time step of $1 \mathrm{fs}$. Each equilibrium simulation consisted of twenty trajectories with different equilibration periods ranging from $0.5 \mathrm{~ns}$ to $0.98 \mathrm{~ns}$; during the first half of each equilibration, velocity rescaling was used to maintain the temperature after which a Nosé-Poincaré thermostat was used. Following equilibration each trajectory was propagated for a $5 \mathrm{~ns}$ data collection period.

The nonequilibrium simulations were initiated with a long equilibration run (1 ns) in the electronic ground state. The molecule was then promoted to the excited state and the fluorescence energy, $\Delta E(t)=E_{e}(t)-E_{g}(t)$, was monitored as a function of time for $200 \mathrm{ps}$; no thermostat was applied to the nonequilibrium dynamics during this period. The system was then returned to its configuration just prior to excitation and propagated in the electronic ground state for $10 \mathrm{ps}$ when the solute molecule was again excited and the TDF data collected. This process was repeated to obtain a total of 1000 nonequilibrium trajectories for each cavity radius. The TDF result can be plotted as the time-dependent Stokes shift, $\langle\Delta E\rangle_{n e}(t)-\langle\Delta E\rangle_{n e}(0)$, or normalized in the usual way as given by $S(t)$ in Eq. (33).

\section{RESULTS AND DISCUSSION}

\section{A. Overview of time-dependent fluorescence in nanoconfined solvents}

Here we provide a brief overview of the general properties of time-dependent fluorescence in nanoconfined solvent systems such as that used here. This will lay the groundwork for the tests of the accuracy of dynamic linear-response and Gaussian statistics approximations discussed below. One of us has shown previously, using Monte Carlo and MD simulations, that nanoconfined solvents exhibit a number of properties that are distinct from the corresponding bulk solvent ${ }^{30,31,41,42}$ and that these can have signatures in simulations of the time-dependent fluorescence. ${ }^{31}$

First, the solvent polarity depends strongly on the distance from the cavity wall. The constraints placed on the solvent molecules near the wall reduce their ability to respond to the charges on a solute molecule in their midst. Molecules near the center of a cavity are not as restricted and can rearrange more freely to accommodate a solute molecule. Thus, the solvent polarity is significantly diminished near the cavity wall and increases moving away from the wall. At the same time, the polarity in the center of the cavity is not that of the bulk for cavities of the size considered here due to the confinement and absence of long-range electrostatic interactions. This position-dependent solvent polarity means that the locations explored by a solute depend on its charge distributions. For example, as shown in Fig. 1 for the $12 \AA$ radius cavity, the $\mathrm{AB}$ dye molecule considered in this work sits near the wall when it is in its ground state but nearer the cavity center when it is in the electronic excited state. Hence, solute diffusion must occur after excitation and, as we have previously demonstrated for this model system, ${ }^{31}$ can be observed in the dynamic Stokes shift.

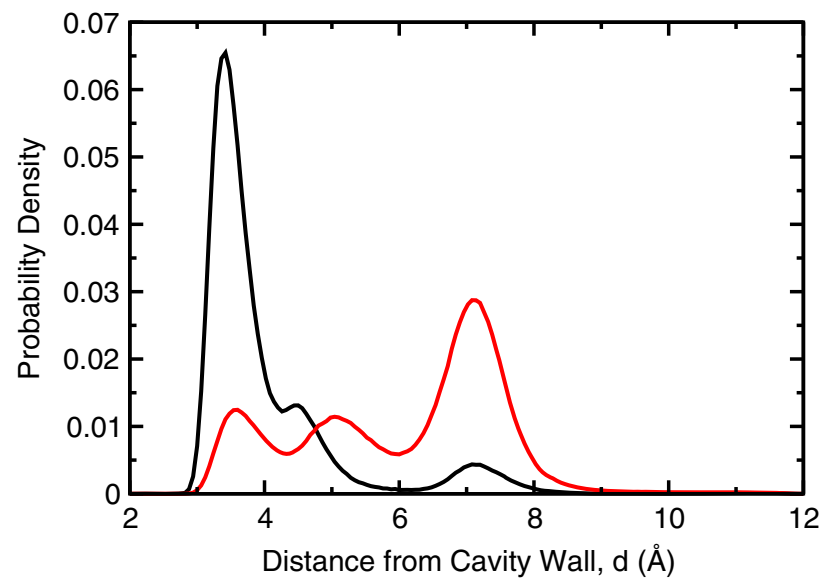

FIG. 1. The radial center-of-mass probability distributions for the AB dye molecule in the ground (black line) and excited (red line) electronic state in $\mathrm{CH}_{3} \mathrm{CN}$ confined within a $12 \AA$ radius spherical cavity.

Second, as with planar solid-liquid interfaces, a nanoconfined solvent forms layers near the surface, exhibiting oscillations in the radial density that decrease in intensity moving away from the cavity wall. These oscillations in the solvent density can govern the position and orientation of a solute molecule in the cavity. ${ }^{30}$ Moreover, the diffusion of a solute in the cavity will take place on a free energy surface that depends on position - with barriers present where the solvent density is low. This can be seen from the structured position distributions of the $\mathrm{AB}$ dye molecule shown in Fig. 1. Specifically, the individual peaks correspond to solute positions within individual solvent layers (which for $\mathrm{CH}_{3} \mathrm{CN}$ means the $\mathrm{AB}$ lies parallel to the cavity wall) or spanning across two solvent layers (where $\mathrm{AB}$ sits perpendicular to the cavity wall). Additional discussion of the underlying free energy surfaces (and the internal energy and entropic contributions) can be found in Ref. 41.

We note that, while the properties of the TDF signal in the model systems examined here are well understood, the picture is less clear for experimental systems. Generally, measurements of TDF reveal additional, longer time scales (as long as several nanoseconds) than observed in the corresponding bulk solvent. While a number of models (including the one presented in Refs. 30 and 31) have been proposed to explain these long time scale components of the TDF signal in nanoconfined solvents, their origin(s) has not been definitively established. However, this is a side issue for the purpose of understanding linear-response and Gaussian statistics approximations, which is the focus of this paper.

\section{B. Tests of linear-response and Gaussian statistics approximations}

\section{Dynamic Stokes shift}

With this background for the model systems in mind, we now turn to direct tests of the linear-response approximations and the assumption of Gaussian statistics described in Sec. II. Figure 2 presents the unnormalized and normalized time-dependent Stokes shifts, $\langle\Delta E(t)\rangle_{n e}$ and $S(t)$, 

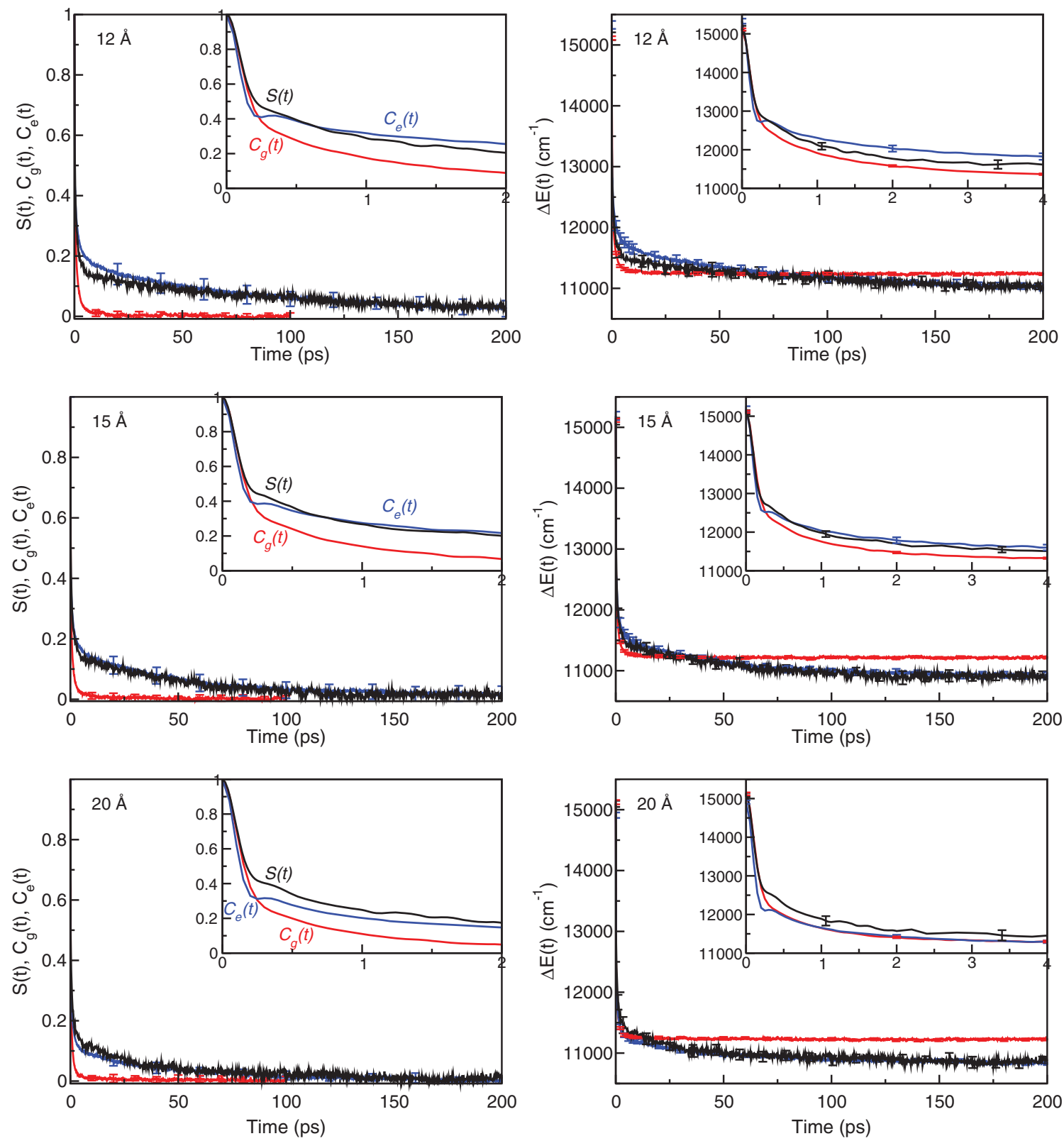

FIG. 2. Dynamic Stokes shifts in normalized and unnormalized form, $S(t)$ and $\langle\Delta E(t)\rangle_{n e}$, respectively, from nonequilibrium MD simulations (black line) are compared with the dynamic linear-response approximation (red line) and an assumption of Gaussian statistics (blue line). Results are shown for a cavity radius of, from top to bottom, 12,15 , and $20 \AA$.

respectively, obtained from the nonequilibrium MD simulations described in Sec. III. Results are shown for spherical cavities of radius 12,15 , and $20 \AA$ and compared with the corresponding linear-response and Gaussian statistics approximations based on equilibrium ground- and excited-state correlation functions, respectively.

We first discuss the general properties of the dynamic Stokes shift, $S(t)$. For all pore sizes considered here, $S(t)$ is reasonably described by a tri-exponential function. The shortest time scale is on the order of $150 \mathrm{fs}$ and represents the inertial response of the system to the change in the $A B$ charge distribution after excitation. The short-time behavior is Gaussian in time, but because it is not of central interest here we will be content with an exponential description. These inertial dynamics represent the largest component of the decay of $S(t)$. The intermediate time scale is $\sim 1.4 \mathrm{ps}$ and is similar to the longest-time component in bulk $\mathrm{CH}_{3} \mathrm{CN}$. Thus, this is presumably due primarily to solvent reorientation; however, we should note that there is solute diffusion, albeit limited, that occurs within the first few picoseconds and thus solvent reorientation and solute diffusion are not uncoupled. ${ }^{31}$ The longest time scale is more than $50 \mathrm{ps}$ and increases with decreasing cavity size. This is related to diffusion of the dye molecule after excitation as discussed above and in Ref. 31; additional details of the connection with solute motion are provided below.

As noted in Sec. II, the dynamic linear-response approximation yields the result $S(t) \simeq C_{g}(t)$. It is clear from Fig. 2 that this is a poor approximation for all three cavity radii considered in this work. In particular, the ground- 
state autocorrelation function for the energy gap, $C_{g}(t)$, decays with only two time scales. Specifically, $C_{g}(t)$ is well described by a bi-exponential function with two time constants that generally agree with the shortest two time scales observed in the decay of $S(t)$. Thus, the dynamic linear-response approximation appears to accurately represent the inertial and solvent reorientation components of the time-dependent fluorescence. On the other hand, the longest time scale observed in $S(t)$, which is associated with solute diffusion, is absent in $C_{g}(t)$. The amplitude associated with this long time scale decay appears to be subsumed into the inertial component in the linear-response approximation; the amplitudes associated with solvent reorientation for $S(t)$ and $C_{g}(t)$, agree reasonably well.

As is shown in the Appendix, $C_{g}(t)=C_{e}(t)$ in the limit of linear response, which is also tested by the data plotted in Fig. 2. Clearly, this linear-response approximation is not supported by these results. In particular, $C_{e}(t)$ agrees well with $S(t)$ and hence differs from $C_{g}(t)$ in that the latter lacks a long-time decay component. More minor differences also exist in the amplitudes and time constants associated with the shorter time scale components. That $C_{g}(t) \neq C_{e}(t)$ should be expected is evident from Fig. 1, which shows the configurations explored by the dye molecule in its ground and excited states are quite different. It is important to note, however, that for this difference to be manifested in the two autocorrelation functions requires that the ground-to-excited-state energy gap depend on the position in the cavity. This is due to the gradient in effective solvent polarity of the confined $\mathrm{CH}_{3} \mathrm{CN}$ discussed above - the increasing solvent polarity with distance from the cavity wall allows the TDF signal to be sensitive to the dyemolecule position. Naturally, it also means that differences in the ground- and excited-state position distributions lead to an inequality between the two gap autocorrelation functions, $C_{g}(t)$ and $C_{e}(t)$.

The assumption that $\Delta E$ exhibits Gaussian statistics yields $S(t)=C_{e}(t)$ and Fig. 2 indicates that this is an excellent approximation for the three cavity sizes considered. In particular, $C_{e}(t)$ is the same as $S(t)$, within error bars, for the entire $200 \mathrm{ps}$ decay for all cases. That $\Delta E$ should be a Gaussian random variable is not obvious a priori for these nanoconfined solvent systems. The gradient in effective solvent polarity within the cavity should lead to a solute positiondependence of the force constant for the free energy surface along the solvent coordinate; this has previously been found for these systems with a $\mathrm{CH}_{3} \mathrm{I}$ solvent ${ }^{30,44,45}$ and is also the case for the $\mathrm{CH}_{3} \mathrm{CN}$ systems examined here. However, the position dependence of the force constant is relatively weak, presumably due to the modest solvent-surface interactions for these systems. Thus, $\Delta E$ does appear to exhibit Gaussian statistics, yet this may not be the case for solvents in more strongly interacting confining frameworks, a topic currently under investigation by one of us.

The results for the unnormalized time-dependent Stokes shift, $\langle\Delta E(t)\rangle_{n e}$, are consistent with those for $S(t)$. It is interesting to note that the assumption of Gaussian statistics for $\Delta E$ yields the correct magnitude for the dynamic Stokes shift as represented by $\langle\Delta E(t)\rangle_{n e}$, a more stringent test than $S(t)$. On the other hand, the dynamic linear-response ap- proximation fails in both cases. For the unnormalized Stokes shift, it predicts a significantly smaller overall change, i.e., $\langle\Delta E(0)\rangle_{n e}-\langle\Delta E(\infty)\rangle_{n e}$, due to its inability to accurately represent the change in solute position after excitation.

\section{Dye-molecule diffusion}

In complex systems such as nanoconfined solvents, the dynamics Stokes shift is not the only property of interest. As discussed above, in the model systems considered here a longtime component appears in the TDF signal that is attributable to diffusion of the chromophore after excitation. Thus, the nonequilibrium change in dye-molecule position is of interest and it is then natural to ask: To what degree do the dynamic linear-response and Gaussian statistics approximations describe this quantity? It is important to re-emphasize in this context the complicated position dependence on electronic state for the dye molecule, as illustrated in Fig. 1 and the corresponding free energy surfaces (see Ref. 41). These suggest that the chromophore position, $d$, should not exhibit Gaussian statistics.

The change in the dye molecule distance from the spherical cavity wall after excitation, obtained from the nonequilibrium MD simulations, is shown for cavities of radius 12 , 15 , and $20 \AA$ in Fig. 3. Also shown are the predictions from the dynamic linear-response approximation and an assumption of Gaussian statistics. While neither approximation is in excellent agreement with the nonequilibrium MD results, the Gaussian statistics result agrees within the error bars for cavities of radii 15 and $20 \AA$, but overestimates $\langle\Delta d(t)\rangle_{n e}$ for $R_{\text {cav }}$ $=12 \AA$. On the other hand, the dynamic linear-response approximation predicts changes in the chromophore position that are significantly too small and, therefore, does not agree well at all with the nonequilibrium MD results. However, we can also examine only the time scales for dye-molecule diffusion, rather than the absolute changes in distance from the cavity wall, by focusing on the unitless quantity $\langle\Delta d(t)\rangle /\langle\Delta d(\infty)\rangle$. In this case, the result is somewhat different: both the dynamic linear-response and Gaussian statistics approximations describe the nonequilibrium MD results within the error bars, which are not small due to the combined uncertainties in $\langle\Delta d(t)\rangle$ and $\langle\Delta d(\infty)\rangle$.

It is worth noting that the total change in the average position can be accurately determined from the averages in the ground and excited states of the long equilibrium MD simulations. This gives $\Delta d(\infty)=2.0,2.5$, and $3.4 \AA$ for cavity radii of 12,15 , and $20 \AA$, respectively. This compares reasonably well to the results from the nonequilibrium MD simulations after $200 \mathrm{ps}$, which yield $\langle\Delta d(200 \mathrm{ps})\rangle_{n e}=1.7 \pm 0.4$, $2.4 \pm 0.2$, and $3.0 \pm 0.5 \AA$ for $R_{\text {cav }}=12,15$, and $20 \AA$. Note that the underestimation of the change in distance for the smallest cavity is partially due to the slower dynamics, i.e., the diffusion is not complete after $200 \mathrm{ps}$. The estimated total change in distance from the dynamic linear-response approximation is clearly too small, as is readily seen from Fig. 3, giving $0.30 \pm 0.07,0.63 \pm 0.10$, and $0.94 \pm 0.13 \AA$ for cavities of radii 12,15 , and $20 \AA$, respectively. The results from an assumption of Gaussian statistics are in better agreement, 

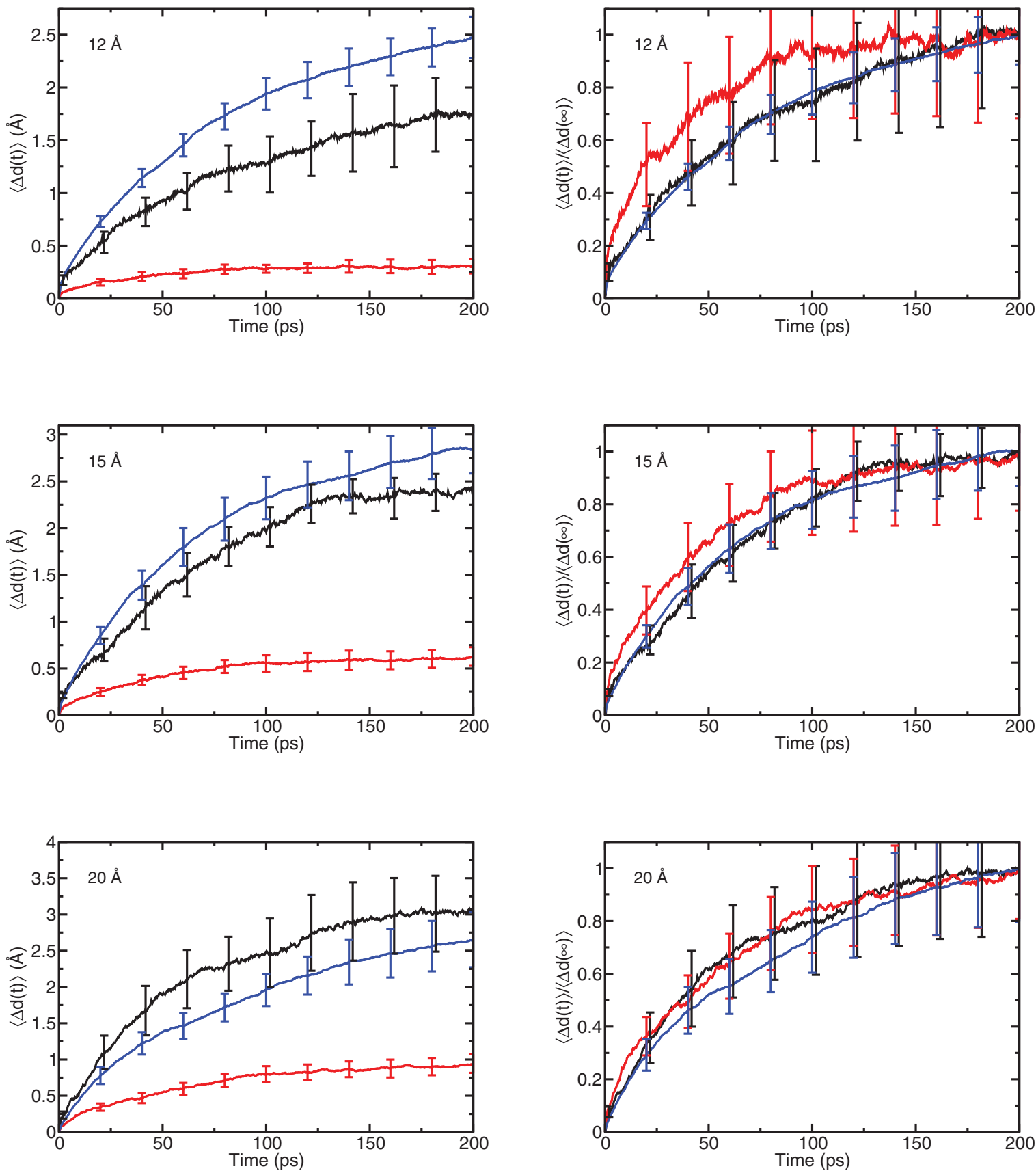

FIG. 3. Change in the chromophore distance from the cavity wall after excitation in unnormalized, $\langle\Delta d(t)\rangle$, (left panels) and normalized, $\langle\Delta d(t)\rangle /\langle\Delta d(\infty)\rangle$, (right panels) form from nonequilibrium MD simulations (black line) are compared with the dynamic linear-response approximation (red line) and an assumption of Gaussian statistics, (blue line). Results are shown for cavity radii of, from top to bottom, 12, 15, and $20 \AA$.

yielding $2.5 \pm 0.2,2.8 \pm 0.3$, and $2.7 \pm 0.4 \AA$, respectively, which agree with the nonequilibrium MD results within error bars for all but the smallest cavity.

The reasonably good agreement between the Gaussian statistics assumption results and the nonequilibrium MD simulations is somewhat surprising given the clearly nonGaussian statistics of the dye-molecule position, $d$ (see Fig. 1). On the other hand, the agreement is distinctly worse than for the dynamic Stokes shift. For example, while $S(t)$ and $\langle\Delta E(t)\rangle_{n e}$ are well described by the Gaussian statistics approximations, $\langle\Delta d(t)\rangle_{n e}$ is not in all cases. This is attributable to the non-Gaussian statistics of $d$. It is interesting, however, that the disagreement is not in the dynamics of $\langle\Delta d(t)\rangle_{n e}$, as $\langle\Delta d(t)\rangle_{n e} /\langle\Delta d(\infty)\rangle_{n e}$ is well-described assuming Gaussian statistics, but in the overall change, $\langle\Delta d(\infty)\rangle_{n e}$.

\section{Static linear-response and Gaussian statistics}

As noted in Sec. II, the static linear-response and Gaussian statistics approximations yield the same results for the quantities of interest in this problem, $\langle\Delta E(t)\rangle_{n e}$ and $\langle\Delta d(t)\rangle_{n e}$. In both approaches these nonequilibrium quantities are expressed in terms of equilibrium excited-state time correlation functions, but the results are arrived at using 

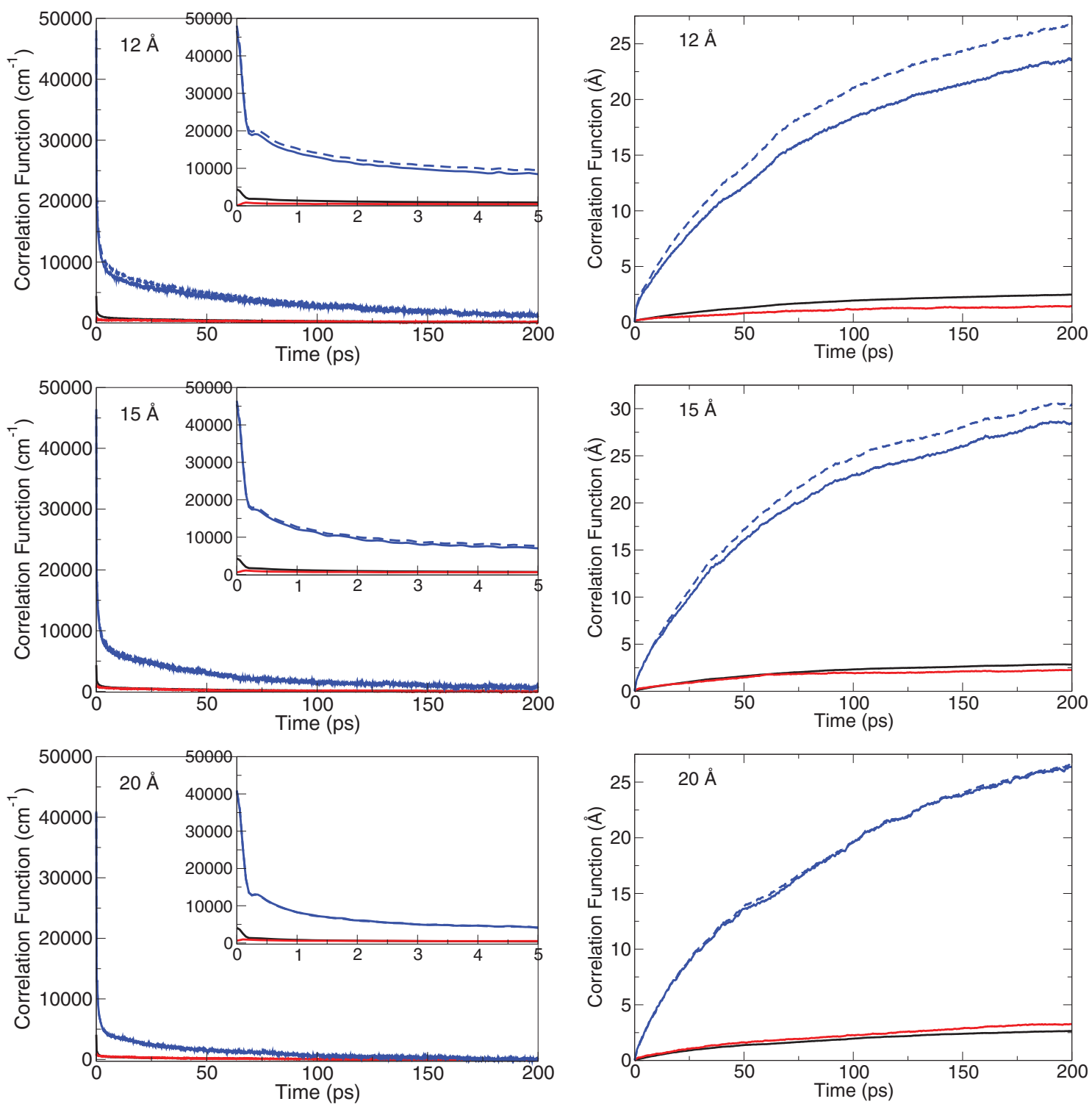

FIG. 4. Higher-order correlation functions are compared for $A=\Delta E$ (left) and $A=d$ (right). Results are shown for $\beta\langle\delta A(t) \delta \Delta E(0)\rangle_{e}$ (solid black line), $\beta^{2}\left\langle\delta A(t) \delta \Delta E(0)^{2}\right\rangle_{e} / 2$ (solid red line), $\beta^{3}\left\langle\delta A(t) \delta \Delta E(0)^{3}\right\rangle_{e} / 6$ (solid blue line), and $\beta^{3}\langle\delta A(t) \delta \Delta E(0)\rangle_{e}\left\langle\delta \Delta E(0)^{2}\right\rangle_{e} / 2$ (dashed blue line). Results are shown for cavity radii of, from top to bottom, 12,15 , and $20 \AA$.

different assumptions in the two cases. It is useful then to explore the validity of these approximations to shed light on which are physically reasonable and to validate our description of the excited-state correlation function results in terms of Gaussian statistics in the above discussion.

One result along these lines was presented above in the comparison of the results from ground- and excited-state correlation functions. Within the static linear-response approximation, these should be equal, as shown in the Appendix. Clearly, from Figs. 2 and 3 one can see that they are not. Further insight can be gained by direct examination of the assumptions in the two theories regarding the higher-order correlation functions. In particular, the approaches diverge in how they approximate the correlation functions in Eq. (17). The static linear-response approach assumes $\left\langle\delta A(t) \delta \Delta E(0)^{n}\right\rangle_{e}=0$ for $n>1$ whereas assuming Gaussian statistics leads to Eqs. (20) and (21), i.e., higher-order correlation functions are not all neg- ligible, but are factorizable. These two approximations can be checked explicitly. For the lowest-order correlation functions at issue, static linear-response takes $\left\langle\delta A(t) \delta \Delta E(0)^{2}\right\rangle_{e}=\left\langle\delta A(t) \delta \Delta E(0)^{3}\right\rangle_{e}=0$, whereas assuming Gaussian statistics yields $\left\langle\delta A(t) \delta \Delta E(0)^{2}\right\rangle_{e}=0$ but $\quad\left\langle\delta A(t) \delta \Delta E(0)^{3}\right\rangle_{e}=3\langle\delta A(t) \delta \Delta E(0)\rangle_{e}\left\langle\delta \Delta E(0)^{2}\right\rangle_{e}$. Moreover, the neglect of these higher-order correlation functions within static linear-response is based on the magnitude in the expansion represented in Eq. (28). That is, $\beta^{2}\left\langle\delta A(t) \delta \Delta E(0)^{2}\right\rangle_{e} / 2 \ll \beta\langle\delta A(t) \delta \Delta E(0)\rangle_{e}$ and $\beta^{3}\left\langle\delta A(t) \delta \Delta E(0)^{3}\right\rangle_{e} / 6 \ll \beta\langle\delta A(t) \delta \Delta E(0)\rangle_{e}$. These functions are compared for both $A=\Delta E$ and $A=d$ in Fig. 4.

The results presented in Fig. 4 clearly show that the mathematical approximation invoked in the static linear-response approach is not justified for either the dynamic Stokes shift, $A=\Delta E$, or the solute diffusion $A=d$. For the Stokes shift, it is the case that $\beta^{2}\left\langle\delta \Delta E(t) \delta \Delta E(0)^{2}\right\rangle_{e} / 2$ is less (if not 
much less) than $\beta\langle\delta \Delta E(t) \delta \Delta E(0)\rangle_{e}$, a result also obtained by assuming Gaussian statistics. However, the higher-order correlation function $\beta^{3}\left\langle\delta A(t) \delta \Delta E(0)^{3}\right\rangle_{e} / 6$ is not at all negligible but rather approximately an order-of-magnitude larger than $\beta\langle\delta \Delta E(t) \delta \Delta E(0)\rangle_{e}$. On the other hand, the factorization provided by Gaussian statistics, Eq. (21), is in excellent agreement with the full correlation function as can be seen by comparing the dashed and solid blue lines in Fig. 4. Thus, for the solvation dynamics in this system, which involves perturbation far from the excited-state equilibrium, the mathematical assumptions of the static linear-response approximation are not valid, but those of Gaussian statistics are. In a sense, this is a positive result for linear response theories in general as Gaussian statistics implies a globally linear response to a perturbation, not merely a local one. It also indicates why the static linear-response approximation has seemed to work well for solvation dynamics ${ }^{32}$ where the assumption of small fluctuations is not valid - the more appropriate viewpoint is that the use of the equilibrium excited-state correlation functions is the invocation of Gaussian statistics. ${ }^{19}$

The results for solute diffusion, $A=d$, differ from the solvation dynamics case primarily in the fact that the correlation function $\beta^{2}\left\langle\delta d(t) \delta \Delta E(0)^{2}\right\rangle_{e} / 2$ is not negligible, which is assumed for both static linear response and Gaussian statistics, but is rather on the same order as $\beta\langle\delta d(t) \delta \Delta E(0)\rangle_{e}$. The remaining differences are slight. It is still the case that $\beta^{3}\left\langle\delta d(t) \delta \Delta E(0)^{3}\right\rangle_{e} / 6$ is roughly an order-of-magnitude larger than $\beta\langle\delta d(t) \delta \Delta E(0)\rangle_{e}$, such that its neglect in the static linear-response approximation is not justified. Further, the factorization obtained by the assumption of Gaussian statistics is still accurate for this case, but introduces greater error than for $A=\Delta E$, particularly as the cavity size decreases. That the approximations are poorer for the solute diffusion is not unexpected given the clearly non-Gaussian properties of the solute position (see Fig. 1).

\section{CONCLUSIONS}

The linear-response and Gaussian statistics approximations have been summarized and applied to the timedependent fluorescence of a model dye molecule in a nanoconfined acetonitrile solvent. While the TDF dynamics is typically probed through the time-dependent Stokes shift, the position of the dye molecule after excitation, an important variable in nanoconfined systems, is also examined here.

The dynamic linear-response approximation fails to fully describe the solvation dynamics in the model nanoconfined solvent system. This is the case for both the normalized and unnormalized dynamic Stokes shift and results from the difference in the position distribution for the dye molecule in its ground and excited electronic states. The narrower position distribution for the ground state leads to an absence of the longest time scale in the Stokes shift dynamics, which is associated with dye molecule diffusion. The agreement is better at shorter times. The dynamic linear-response approximation, $C_{g}(t)$, describes the $\sim 150 \mathrm{fs}$ inertial solvation dynamics quite well, consistent with previous reports for other models $9,14,15$ and attributed to the similarity between the nonequilibrium inertial motion and the equilibrium dynamics in the ground state. An intermediate time scale in the solvation dynamics corresponding to diffusive solvent reorientation, is also reasonably represented in the linear response results, though its amplitude is not. This component is similar to the longest time scale observed in the unconfined liquid and the agreement indicates that the bulk solvent is accurately described by the dynamic linear-response approximation. These results suggest that, to the extent this model is representative of real systems, nanoconfined solvents may represent experimentally accessible systems that exhibit violations of the dynamic linearresponse approximation.

While the dynamic linear-response approach is based on ground-state equilibrium dynamics, one can instead use correlation functions obtained from equilibrium excited-state dynamics. Both the static linear-response and Gaussian statistics approximations, applied to the nonequilibrium dynamics, yield excited-state correlation functions, but based on different approximations. Static linear response assumes all higher-order correlation functions are negligible, while Gaussian statistics factorizes them into lower-order correlation functions. Because the final expressions for nonequilibrium quantities are identical in both approaches, we have examined the underlying approximations to investigate which are justified (and, hence, which physical picture is appropriate for the mathematical approximation). We have found that the higher-order correlation functions are far from negligible but can be accurately factored. Further, the static linear-response approximation predicts that the ground- and excited-state dynamics should yield the same result (see the Appendix), which is clearly not the case. These results thus indicate that the approximation of the nonequilibrium response by equilibrium excited-state correlation functions is best viewed within the assumption of Gaussian statistics, not static linear-response. This indicates that the approximation assumes response that is globally linear and not merely valid for small fluctuations away from the excited-state equilibrium (as required in the static linear-response approach).

If one assumes that the energy gap, $\Delta E$, exhibits Gaussian statistics, excellent agreement with the nonequilibrium MD results is obtained. This is true for the normalized and unnormalized dynamics Stokes shift and for all of the observed time scales and amplitudes. Thus, while the dynamic linear-response approximation reproduces the inertial component of the solvation dynamics, the agreement with the Gaussian statistics result is also quite reasonable. The major difference between the results from the two approximations is the long-time ( $>50 \mathrm{ps}$ ) dynamics associated with the diffusion of the solute dye molecule after excitation. This feature in the solvation dynamics is fully reproduced by assuming $\Delta E$ is a Gaussian random variable, but not at all by the dynamic linear-response approximation. In one sense, this is an illustration of the general (but not universal) result found in the literature that the excited-state equilibrium dynamics represent the nonequilibrium response better than the groundstate dynamics. However, it is important to note that the equilibrium correlation functions in the two states are arrived at from different approximations. Thus, this result can also be understood as an indication that the assumption of Gaussian 
statistics is a distinctly different and less severe approximation than that of dynamic linear response.

At the same time, it is somewhat surprising that even the Gaussian statistics approximation would work for this case. It was previously found that for the same nanoconfined system with methyl iodide as the solvent instead of acetonitrile, the free energy as a function of $\Delta E$ is harmonic for a fixed dye-molecule position. ${ }^{30}$ However, the force constant for the free energy surface depends on the position. ${ }^{44,45}$ Thus, as the dye molecule diffuses after excitation, the statistics are Gaussian, but nonstationary, the same situation which gives $C_{e}(t) \neq S(t)$ for the dipole-flip dye model. ${ }^{14,15,19}$ Presumably, the changes in the Gaussian statistics of $\Delta E$ with position in the nanocavity are then comparatively modest and are not sufficient to lead to significant disagreement with the nonequilibrium MD results. It may be that in nanoconfining frameworks with stronger solvent-surface interactions this will not be the case and the Gaussian statistics approximation will fail to describe the TDF solvation dynamics.

The results are similar for the change in the dye molecule distance from the cavity wall after excitation. The dynamic linear-response approximation fails to describe the significant change in solute position as the ground state distribution of positions is significantly narrower than that in the excited state. On the other hand, the assumption of Gaussian statistics gives good agreement with the nonequilibrium MD results for all but the smallest cavity. That reasonable agreement is obtained at all is unexpected, as the solute position, $d$, should not exhibit Gaussian statistics (see Fig. 1), though the driving force for the transition, $\Delta E$ nearly does so. As in the case of solvation dynamics, this suggests that the degree of nonGaussian behavior is important and satisfactory results can be obtained as long as the deviation from Gaussian statistics is not too great. The degree to which this applies in other contexts is worth exploring.

\section{ACKNOWLEDGMENTS}

B.B.L. gratefully acknowledges support from the National Science Foundation (NSF) under Grant No. CHE0957102. W.H.T. thanks the Chemical Sciences, Geosciences and Biosciences Division, Office of Basic Energy Sciences, Office of Science, (U.S.) Department of Energy (DOE) for support of this work.

\section{APPENDIX: EQUIVALENCE OF DYNAMIC AND STATIC LINEAR-RESPONSE APPROXIMATIONS}

In the paper, we have two equations for $\langle A(t)\rangle_{n e}$ within the dynamic and static linear-response theories. The dynamic linear-response result, based on dynamics on the ground state, is given in Eq. (13) and the static linear-response expression, based on dynamics in the excited state, is provided in Eq. (26). Here we show that these are identical in the linear-response limit. That is, their difference is second-order in the perturbation $\Delta E$. We do this by expanding Eq. (26) about the ground state,

$$
\begin{aligned}
\langle A(t)\rangle_{s}= & \langle A\rangle_{e}+\beta\left[\langle A(t) \Delta E(0)\rangle_{e}-\langle A\rangle_{e}\langle\Delta E(0)\rangle_{e}\right] \\
= & \frac{\operatorname{Tr}\left[A e^{-\beta H_{e}}\right]}{\operatorname{Tr}\left[e^{-\beta H_{e}}\right]}+\frac{\beta \operatorname{Tr}\left[e^{\hat{L}_{e} t} A(0) \Delta H(0) e^{-\beta H_{e}}\right]}{\operatorname{Tr}\left[e^{-\beta H_{e}}\right]} \\
& -\frac{\beta \operatorname{Tr}\left[A e^{-\beta H_{e}}\right]}{\operatorname{Tr}\left[e^{-\beta H_{e}}\right]} \frac{\operatorname{Tr}\left[\Delta H(0) e^{-\beta H_{e}}\right]}{\operatorname{Tr}\left[e^{-\beta H_{e}}\right]}
\end{aligned}
$$

The three terms in Eq. (A1) can be examined separately. First,

$$
\begin{aligned}
\frac{\operatorname{Tr}\left[A e^{-\beta H_{e}}\right]}{\operatorname{Tr}\left[e^{-\beta H_{e}}\right]}= & \frac{\operatorname{Tr}\left[A e^{-\beta\left(H_{g}+\Delta H\right)}\right]}{\operatorname{Tr}\left[e^{\left.-\beta H_{g}+\Delta H\right)}\right]} \\
= & \frac{\operatorname{Tr}\left[A e^{-\beta H_{g}}(1-\beta \Delta H)\right]}{\operatorname{Tr}\left[e^{-\beta H_{g}}(1-\beta \Delta H)\right]}+\mathcal{O}\left[(\Delta E)^{2}\right] \\
= & \frac{Q_{g}\langle A\rangle_{g}-\beta Q_{g}\langle\Delta E\rangle_{g}}{Q_{g}-Q_{g}\langle\Delta E\rangle_{g}}+\mathcal{O}\left[(\Delta E)^{2}\right] \\
= & \frac{\langle A\rangle_{g}-\beta\langle\Delta E\rangle_{g}}{1-\langle\Delta E\rangle_{g}}+\mathcal{O}\left[(\Delta E)^{2}\right] \\
= & \left.\left(\langle A\rangle_{g}-\beta\langle\Delta E\rangle_{g}\right)\left(1+\langle\Delta E\rangle_{g}\right)\right) \\
& +\mathcal{O}\left[(\Delta E)^{2}\right] \\
\frac{\operatorname{Tr}\left[A e^{-\beta H_{e}}\right]}{\operatorname{Tr}\left[e^{-\beta H_{e}}\right]}= & \langle A\rangle_{g}-\beta\langle A(0) \Delta E(0)\rangle_{g}+\beta\langle A\rangle_{g}\langle\Delta E\rangle_{g} \\
& +\mathcal{O}\left[(\Delta E)^{2}\right] .
\end{aligned}
$$

The second term in Eq. (A1) is

$$
\begin{aligned}
\frac{\operatorname{Tr}\left[e^{\hat{L}_{e} t} A(0) \Delta H(0) e^{-\beta H_{e}}\right]}{\operatorname{Tr}\left[e^{-\beta H_{e}}\right]} & \frac{\operatorname{Tr}\left[e^{\left(\hat{L}_{g}+\Delta \hat{L}\right) t} A(0) \Delta H(0) e^{-\beta\left(H_{g}+\Delta H\right)}\right]}{\operatorname{Tr}\left[e^{-\beta\left(H_{g}+\Delta H\right)}\right]} \\
= & \left(1+\beta\langle\Delta H\rangle_{g}+\cdots\right) \frac{1}{Q_{g}} \operatorname{Tr}\left[e^{\hat{L}_{g} t}\right. \\
& \times\left(1+\int_{0}^{t} d \tau e^{-\hat{L}_{g} \tau} \Delta \hat{L} e^{\hat{L}_{g} \tau}+\cdots\right) \\
& \left.\times A(0) \Delta H(0) e^{-\beta H_{g}}(1-\beta \Delta H+\cdots)\right] \\
= & \frac{\operatorname{Tr}\left[e^{\hat{L}_{g} t} A(0) \Delta H(0) e^{-\beta H_{g}}\right]}{\operatorname{Tr}\left[e^{-\beta H_{g}}\right]}+\mathcal{O}\left[(\Delta E)^{2}\right] \\
& \frac{\operatorname{Tr}\left[e^{\hat{L}_{e} t} A(0) \Delta H(0) e^{-\beta H_{e}}\right]}{\operatorname{Tr}\left[e^{-\beta H_{e}}\right]} \\
= & \langle A(t) \Delta E(0)\rangle_{g}+\mathcal{O}\left[(\Delta E)^{2}\right] .
\end{aligned}
$$

Finally, the third term in Eq. (A1), we have

$$
\begin{aligned}
& \frac{\operatorname{Tr}\left[A e^{-\beta H_{e}}\right]}{\operatorname{Tr}\left[e^{-\beta H_{e}}\right]} \frac{\operatorname{Tr}\left[\Delta H(0) e^{-\beta H_{e}}\right]}{\operatorname{Tr}\left[e^{-\beta H_{e}}\right]} \\
& =\left[\langle A\rangle_{g}-\beta\langle A(0) \Delta E(0)\rangle_{g}+\beta\langle A\rangle_{g}\langle\Delta E\rangle_{g}\right] \\
& \times\left[\langle\Delta E\rangle_{g}-\beta\left\langle[\Delta E(0)]^{2}\right\rangle_{g}+\beta\langle\Delta E\rangle_{g}^{2}\right]+\mathcal{O}\left[(\Delta E)^{2}\right] \\
& =\langle A\rangle_{g}\langle\Delta E\rangle_{g}+\mathcal{O}\left[(\Delta E)^{2}\right] .
\end{aligned}
$$

Combining these results gives 


$$
\begin{aligned}
\langle A(t)\rangle_{s}= & \langle A\rangle_{g}-\beta\langle A(0) \Delta E(0)\rangle_{g}+\beta\langle A\rangle_{g}\langle\Delta E\rangle_{g} \\
& +\beta\langle A(t) \Delta E(0)\rangle_{g}-\beta\langle A\rangle_{g}\langle\Delta E\rangle_{g}+\mathcal{O}\left[(\Delta E)^{2}\right] \\
= & \langle A\rangle_{g}+\beta\left[\langle A(t) \Delta E(0)\rangle_{g}-\langle A(0) \Delta E(0)\rangle_{g}\right] \\
& +\mathcal{O}\left[(\Delta E)^{2}\right] \\
\langle A(t)\rangle_{s}= & \langle A(t)\rangle_{d}+\mathcal{O}\left[(\Delta E)^{2}\right],
\end{aligned}
$$

so that within the linear-response limit in which $\mathcal{O}\left[(\Delta E)^{2}\right]$ terms are neglected, the two approximations are the same.

${ }^{1}$ E. R. Barthel, I. B. Martini, and B. J. Schwartz, J. Phys. Chem. B 105, 12230 (2001).

${ }^{2}$ M. J. Bedard-Hearn, R. E. Larsen, and B. J. Schwartz, J. Phys. Chem. A 107, 4773 (2003).

${ }^{3}$ A. E. Bragg, M. C. Cavanagh, and B. J. Schwartz, Science 321, 1817 (2008).

${ }^{4}$ M. C. Larsen and B. J. Schwartz, J. Chem. Phys. 131, 154506 (2009).

${ }^{5}$ W. J. Glover, R. E. Larsen, and B. J. Schwartz, J. Chem. Phys. 132, 144102 (2010).

${ }^{6}$ A. E. Bragg, W. J. Glover, and B. J. Schwartz, Phys. Rev. Lett. 104, 233005 (2010).

${ }^{7}$ R. M. Stratt, Science 321, 1789 (2008).

${ }^{8}$ A. C. Moskun, A. E. Jailaubekov, S. E. Bradforth, G. H. Tao, and R. M. Stratt, Science 311, 1907 (2006).

${ }^{9}$ G. Tao and R. M. Stratt, J. Chem. Phys. 125, 114501 (2006).

${ }^{10}$ B. H. Savitzky and R. M. Stratt, J. Phys. Chem. B 112, 13326 (2008).

${ }^{11}$ R. Kubo, M. Toda, and N. Hashitsume, Statistical Physics II. Nonequilibrium Statistical Mechanics (Springer-Verlag, New York, 1978).

${ }^{12}$ J.-P. Hansen and I. R. McDonald, Theory of Simple Liquids (Academic, San Diego, CA, 1986).

${ }^{13}$ R. Zwanzig, Nonequilibrium Statistical Mechanics (Oxford University Press, New York, 2001).

${ }^{14}$ T. Fonseca and B. M. Ladanyi, J. Phys. Chem. 95, 2116 (1991).

${ }^{15}$ T. Fonseca and B. M. Ladanyi, J. Mol. Liq. 60, 1 (1994).

${ }^{16}$ M. S. Skaf and B. M. Ladanyi, J. Phys. Chem. 100, 18258 (1996)

${ }^{17}$ V. Tran and B. J. Schwartz, J. Phys. Chem. B 103, 5570 (1999).

${ }^{18}$ D. Aherne, V. Tran, and B. J. Schwartz, J. Phys. Chem. B 104, 5382 (2000).
${ }^{19}$ P. L. Geissler and D. Chandler, J. Chem. Phys. 113, 9759 (2000).

${ }^{20}$ B. B. Laird and W. H. Thompson, J. Chem. Phys. 126, 211104 (2007).

${ }^{21}$ D. Chandler, Introduction to Modern Statistical Mechanics (Oxford University Press, New York, 1987).

${ }^{22}$ L. Turi, P. Minary, and P. J. Rossky, Chem. Phys. Lett. 316, 465 (2000).

${ }^{23}$ D. A. McQuarrie, Statistical Mechanics (Harper, New York, 1976).

${ }^{24}$ M. C. Wang and G. E. Uhlenbeck, Rev. Mod. Phys. 17, 323 (1945).

${ }^{25}$ G. C. Wick, Phys. Rev. 80, 268 (1950).

${ }^{26}$ E. A. Carter and J. T. Hynes, J. Chem. Phys. 94, 5961 (1991).

${ }^{27}$ L. Onsager, Phys. Rev. 37, 405 (1931).

${ }^{28}$ L. Onsager, Phys. Rev. 38, 2265 (1931).

${ }^{29}$ W. Bernard and H. B. Callen, Rev. Mod. Phys. 31, 1017 (1959).

${ }^{30}$ W. H. Thompson, J. Chem. Phys. 117, 6618 (2002).

${ }^{31}$ W. H. Thompson, J. Chem. Phys. 120, 8125 (2004).

${ }^{32}$ M. Maroncelli, J. Chem. Phys. 94, 2084 (1991).

${ }^{33} \mathrm{~A}$. Warshel, Computer Modelling of Chemical Reactions in Proteins and Solution (Wiley, New York, 1991).

${ }^{34} \mathrm{~W}$. L. Jorgensen and J. M. Briggs, Mol. Phys. 63, 547 (1988).

${ }^{35}$ P. Linse, J. Chem. Phys. 90, 4992 (1989).

${ }^{36}$ J. Faeder and B. M. Ladanyi, J. Phys. Chem. B 104, 1033 (2000).

${ }^{37}$ R. C. Weast, editor, Handboook of Chemistry and Physics, 52nd ed. (The Chemical Rubber Company, Cleveland, OH, 1971).

${ }^{38}$ The actual densities may be slightly less than these nominal densities because for a fixed cavity size it is not possible to attain an arbitrary density. The density is calculated by reducing the nominal cavity radius by $0.5 \sigma_{\text {wall }}$ ( $\sigma_{\text {wall }}$ is the effective Lennard-Jones radius of the cavity wall) to approximately account for the excluded volume, a quantity that changes significantly with cavity size.

${ }^{39}$ S. D. Bond, B. J. Leimkuhler, and B. B. Laird, J. Comput. Phys. 151, 114 (1999).

${ }^{40}$ J. M. Sanz-Serna and M. P. Calvo, Numerical Hamiltonian Problems (Chapman and Hall, New York, 1994).

${ }^{41}$ K. R. Mitchell-Koch and W. H. Thompson, J. Phys. Chem. C 111, 11991 (2007).

${ }^{42}$ W. H. Thompson, Annu. Rev. Phys. Chem. 62, 599 (2011).

${ }^{43}$ A. Nitzan, Chemical Dynamics in Condensed Phases (Oxford University Press, New York, 2006).

${ }^{44}$ We note that in Ref. 30 the force constants were incorrectly reported to be different in the ground and excited state for one of the dye molecule positions. In fact they must be identical, as shown in Ref. 45.

${ }^{45}$ M. Tachiya, J. Phys. Chem. 93, 7050 (1989). 